OPEN ACCESS

Edited by:

Haiyang Tang,

University of Arizona, United States

Reviewed by:

Pritesh Jain,

University of California, San Diego,

United States

Tong Zhou,

University of Nevada, Reno,

United States

*Correspondence:

Yuelan Wang

wyldgf_qy@163.com

tThese authors have contributed equally to this work

Specialty section

This article was submitted to

Vascular Physiology,

a section of the journal

Frontiers in Physiology

Received: 19 January 2021

Accepted: 16 February 2021

Published: 01 April 2021

Citation:

Wang D, Dai C, Zhang X, Gu C, Liu M,

$L i u H$, Yang $F$, Wu $H$ and Wang $Y$

(2021) Identification and Functional Analysis of Long Non-coding RNAs in Human Pulmonary Microvascular

Endothelial Cells Subjected to Cyclic Stretch. Front. Physiol. 12:655971. doi: 10.3389/fphys.2021.655971

\section{Identification and Functional Analysis of Long Non-coding RNAs in Human Pulmonary Microvascular Endothelial Cells Subjected to Cyclic Stretch}

\author{
Dong Wang ${ }^{1+}$, Chenyang Dai ${ }^{2 \dagger}$, Xiaoning Zhang ${ }^{3}$, Changping Gu ${ }^{1}$, Mengjie Liu ${ }^{1}$, \\ Huan Liu $^{3}$, Fan Yang ${ }^{1}$, Haifeng $W u^{1}$ and Yuelan Wang ${ }^{1,3 *}$ \\ ${ }^{1}$ Department of Anesthesiology, The First Affiliated Hospital of Shandong First Medical University \& Shandong Provincial \\ Qianfoshan Hospital, Shandong Institute of Anesthesia and Respiratory Critical Medicine, Jinan, China, ${ }^{2}$ Department of \\ Ophthalmology, Qilu Hospital of Shandong University, Jinan, China, ${ }^{3}$ Department of Anesthesiology, Shandong Provincial \\ Qianfoshan Hospital, Shandong University, Cheeloo College of Medicine, Jinan, China
}

Background: Despite decades of intense research, the pathophysiology and pathogenesis of acute respiratory distress syndrome (ARDS) are not adequately elucidated, which hamper the improvement of effective and convincing therapies for ARDS patients. Mechanical ventilation remains to be one of the primary supportive approaches for managing ARDS cases. Nevertheless, mechanical ventilation leads to the induction of further aggravating lung injury which is known as leading to ventilator-induced lung injury (VILI). It has been reported that IncRNAs play important roles in various cellular process through transcriptional, posttranscriptional, translational, and epigenetic regulations. However, to our knowledge, there is no investigation of the expression profile and functions of transcriptome-level endothelium-related IncRNAs in VILI yet.

Methods: To screen the differential expression of IncRNAs and mRNAs in Human pulmonary microvascular endothelial cells (HPMECs) subjected to cyclic stretch, we constructed a cellular model of VILI, followed by transcriptome profiling using Affymetrix Human Transcriptome Array 2.0. Bioinformatics analyses, including functional and pathway enrichment analysis, protein-protein interaction network, IncRNA-mRNA coexpression network, and cis-analyses, were performed to reveal the potential functions and underlying mechanisms of differentially expressed IncRNAs.

Results: In total, 199 differentially expressed IncRNAs (DELs) and 97 differential expressed mRNAs were screened in HPMECs subjected to $20 \%$ cyclic stretch for $2 \mathrm{~h}$. The IncRNA-mRNA coexpression network suggested that DELs mainly enriched in response to hypoxia, response to oxidative stress, inflammatory response, cellular response to hypoxia, and NF-kappa B signaling pathway. LncRNA n335470, n406639, n333984, and n337322 might regulate inflammation and fibrosis induced by cyclic stretch through cis- or trans-acting mechanisms. 
Conclusion: This study provides the first transcriptomic landscape of differentially expressed IncRNAs in HPMECs subjected to cyclic stretch, which provides novel insights into the molecular mechanisms and potential directions for future basic and clinical research of VILI.

Keywords: IncRNA, expression profile, cyclic stretch, human pulmonary microvascular endothelial cell, MMP1, TNFAIP3, TLR4

\section{INTRODUCTION}

Acute respiratory distress syndrome (ARDS) is a life-threatening clinical syndrome characterized by non-cardiogenic pulmonary edema and severe hypoxemia. The common etiologies of ARDS are sepsis, pneumonia, and aspiration of gastric contents. Recent studies demonstrated a progressive decline of the incidence of ARDS, accounting for $10 \%$ in the intensive care unit globally. However, the mortality of ARDS was still as high as $30-40 \%$ in many countries (Bellani et al., 2016; Pham and Rubenfeld, 2017; Matthay et al., 2019). Despite decades of intense research, the pathophysiology and pathogenesis of ARDS are not adequately elucidated, which hamper the improvement of effective and convincing therapies for ARDS patients. Mechanical ventilation remains to be one of the primary supportive approaches for managing ARDS cases (Fan et al., 2018; Matthay et al., 2020). Nevertheless, mechanical ventilation leads to the induction of further aggravate lung injury which is known as leading to ventilator-induced lung injury (VILI) (Moraes et al., 2014; Bates and Smith, 2018). However, the underlying mechanisms of endothelial dysfunction in VILI are still unclear.

Deciphering transcriptional regulation are critical for understanding the molecular mechanisms of majority of biological processes. It has been suggested that lncRNAs, which comprised the majority of transcriptome, play important roles in various cellular process through transcriptional, posttranscriptional, translational, and epigenetic mechanisms (Rinn and Chang, 2012; Batista and Chang, 2013; Quinn and Chang, 2016; Tao et al., 2016). Recent studies have demonstrated that lncRNA MALAT1, PRNCR1, THRIL, and CASC9, acting as a competitive endogenous RNA, played key regulatory roles in sepsis-induced acute lung injury (Chen et al., 2020; Nan et al., 2020; Wang et al., 2020; Yu et al., 2020). It has been proposed that NEAT1 and $\operatorname{lncRNA-5657}$ provided attractive targets for developing therapeutic strategy for ARDS (Liu et al., 2020; Zhou et al., 2020). To our knowledge, there is no investigation study of the expression profile and functions of transcriptome-level endothelium-related lncRNAs in VILI yet.

In the study, we used the Affymetrix Human Transcriptome Array 2.0 to screen differentially expressed IncRNAs and mRNAs in HPMECs subjected to cyclic stretch. Bioinformatics analyses, including functional and pathway enrichment analysis, protein-protein interaction network, cis-analyses of lncRNA, and IncRNA-mRNA coexpression network, were performed to explore the potential functions and underlying mechanisms of differentially expressed lncRNAs.
The findings provide new insights into the molecular regulatory mechanisms of IncRNAs in HPMECs exposed to cyclic stretch, and might contribute to the discovery of new therapeutic targets.

\section{MATERIALS AND METHODS Cell Culture and Cyclic Stretch}

Human pulmonary microvascular endothelial cells (HPMECs, ScienCell, San Diego, CA, USA) were cultured according to the manufacturer's instructions. Based on previous literature (Abiko et al., 2015; Meliton et al., 2015; Tian et al., 2016), HPMECs were subjected to $20 \%$ cyclic stretch for 0 or $2 \mathrm{~h}$ using the FX5000T Flexercell Tension Plus system (Flexcell International, McKeesport, PA) ( $n=3$ per group).

\section{RNA Extraction and Microarray Hybridization}

HPMECs were harvested for RNA extraction using Trizol reagent (Thermo Fisher Scientific, Waltham, MA, United States). RNA quantification and purity were determined using a Nanodrop ND-2000. Then, cDNA labeling and microarray hybridization were performed by the Genminix Informatics Company (Shanghai, China).

\section{Differential Expression Analysis}

GCBI (https://www.gcbi.com.cn), which is a comprehensive bioinformatics analysis platform, provides data analysis online based on $\mathrm{R}$ programming language. The microarray data were pre-processed using the Robust Multi-array Average (RMA) method for background-corrected, normalization, and summary on the GCBI bioinformatics platform (Kong et al., 2016; Huang et al., 2018). Subsequently, Significant Analysis of Microarray (SAM) algorithm was used to identify differentially expressed lncRNAs (DELs) and differentially expressed genes (DEGs) with the fold change cutoff of 1.2 and $p$-value cutoff of 0.05 according to the prior reported literatures (Jia and Zhai, 2019; Li et al., 2020; Zhang et al., 2020). Hierarchical clustering was performed to distinguish the different gene clustering patterns on the same platform.

\section{Functional and Pathway Enrichment Analysis}

Kyoto Encyclopedia of Genes and Genomes (KEGG) pathway and Gene Ontology (GO) functional enrichment analysis were performed using The Database for Annotation, Visualization, and Integrated Discovery (DAVID v6.8, https://david.ncifcrf.gov) 

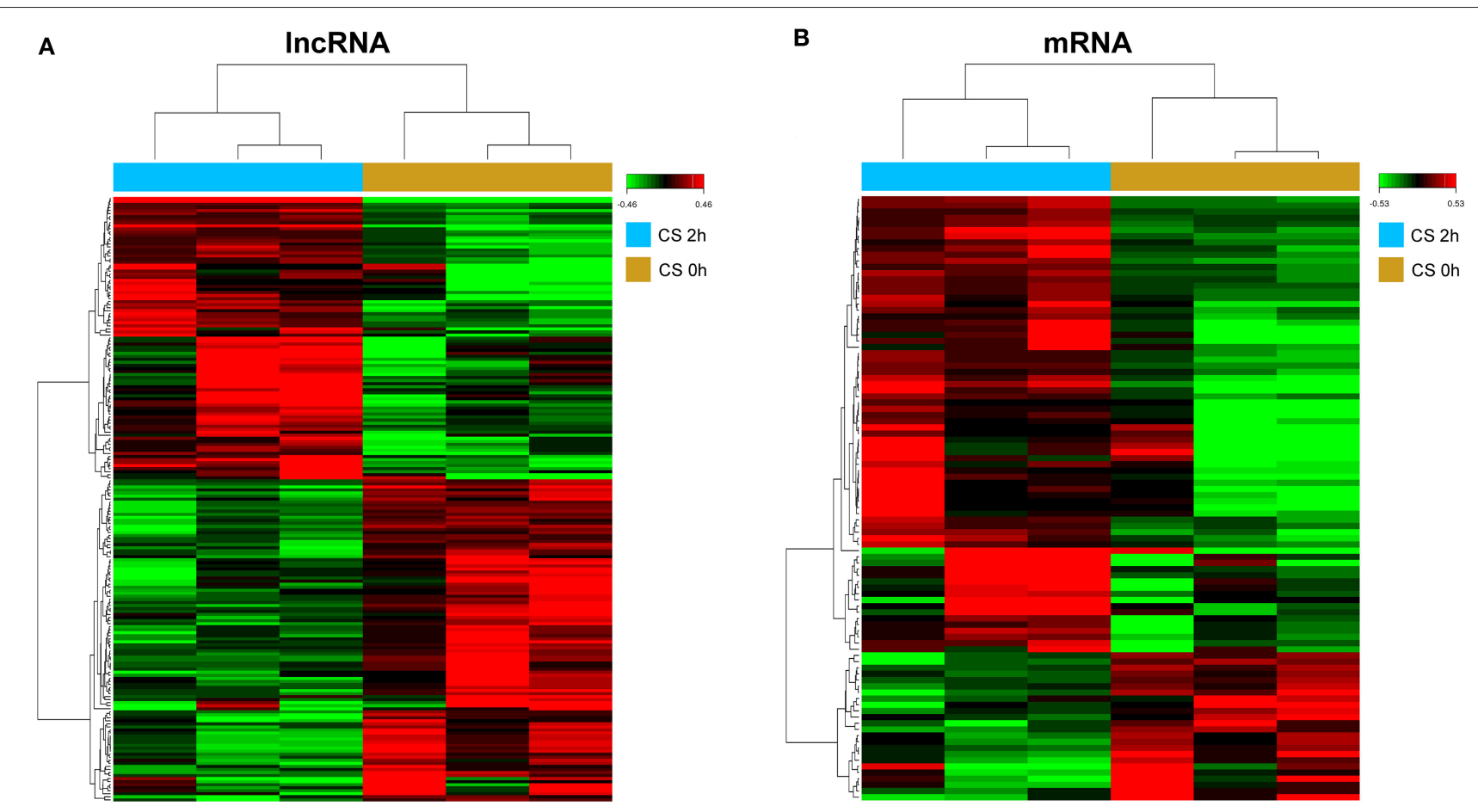

FIGURE 1 | Hierarchical clustering of differentially expressed IncRNAs (DELs) (A) and differentially expressed genes (DEGs) (B). Red to green colors refer to be high to low relative expression levels.

(Huang da et al., 2009a,b; Jiao et al., 2012). GO includes three items: cellular component, molecular function, and biological process. $P<0.05$ was considered to be statistically significant.

\section{Protein-Protein Interaction Network Construction and Module Analysis}

PPI network was constructed in the Search Tool for the Retrieval of Interacting Genes/ Proteins database (STRING v11.0, https:// string-db.org) with minimum required interaction score set in 0.4 (Szklarczyk et al., 2019), and visualized in the Cytoscape (V3.8.2) subsequently (Shannon et al., 2003; Otasek et al., 2019). Module analysis of PPI network was conducted by the MCODE application in Cytoscape, with node score cut off $>3.0$ (Bader and Hogue, 2003).

\section{LncRNA-mRNA Coexpression Network}

LncRNA-mRNA coexpression network was constructed in the GCBI online analysis tool and visualized in the Cytoscape (V3.8.2), subsequently. The functional enrichment analysis of the DEGs in the lncRNA-mRNA coexpression network was performed by the DAVID online tool with a significance threshold of $p<0.05$.

\section{Cis-Analyses of Differentially Expressed IncRNAs}

UCSC Genome Bioinformatics tool (http://genome.ucsc.edu) was used to classify lncRNA cis-target genes. We searched genes within 10k upstream and downstream of DELs, and found the adjacent mRNAs and then analyzed their differential expression.

\section{RESULTS}

\section{Identification of Differentially Expressed IncRNAs and mRNAs}

To screen the differential expression of mRNAs and lncRNAs in HPMECs subjected to cyclic stretch, we performed highthroughput analysis using the Affymetrix Human Transcriptome Array 2.0. After data preprocessing, a total of $199 \operatorname{lncRNAs}$ were determined to be differentially expressed (Figure 1A; Supplementary Material 1). Among these lncRNAs, 93 lncRNAs were upregulated and $106 \mathrm{lncRNAs}$ were downregulated. At the same time, 97 mRNAs were significantly differentially expressed (Figure 1B; Supplementary Material 2), including 74 upregulated mRNAs and 23 downregulated mRNAs.

\section{GO Functional and KEGG Pathway Enrichment Analysis}

To explore the biological processes and pathways of DEGs in HPMECs exposed to cyclic stretch, GO functional and KEGG pathway enrichment analyses were performed. KEGG pathway enrichment analysis revealed that the NF-kappa $\mathrm{B}$ signaling pathway was the most significantly enriched pathway (Figure 2A). GO functional enrichment analysis showed that DEGs were mainly enriched in biological processes (BP) of oxidation-reduction process, inflammatory response, 




angiogenesis, response to hypoxia, cytokine-mediated signaling pathway, response to oxidative stress, and cellular response to mechanical stimulus (Figure 2B). As for cell component (CC), DEGs showed enrichment in plasma membrane, extracellular space, extracellular region, integral component of plasma membrane, and cell surface (Figure 2C). Besides, molecular function (MF) analysis indicated enrichment predominantly at cytokine activity and transcription cofactor activity (Figure 2D).

\section{PPI Network Construction, Module Analysis, and Hub Gene Selection}

To construct the protein-protein interaction network and identify hub genes in HPMECs exposed to cyclic stretch, STRING database was used to predict the interactions of DEGs, and Cytoscape was used for visualization. For the 97 DEGs, the PPI network contained 65 nodes and 140 edges (Figure 3 ). The top nine most significantly hub genes were PTGS2, MMP1, IL1A, TLR4, TNFAIP3, EGR1, HMOXQ, CYP1A1, and PLAU.

\section{LncRNA-mRNA Coexpression Networks}

To address the potential functions of DELs in HPMECs subjected to cyclic stretch, we built a lncRNA-mRNA coexpression network that contained 149 DELs, 82 interacting DEGs, and 140 interaction pairs (Figure 4). KEGG pathway enrichment analysis demonstrated that the Metabolic pathways and NFkappa B signaling pathway were significantly enriched, as shown 


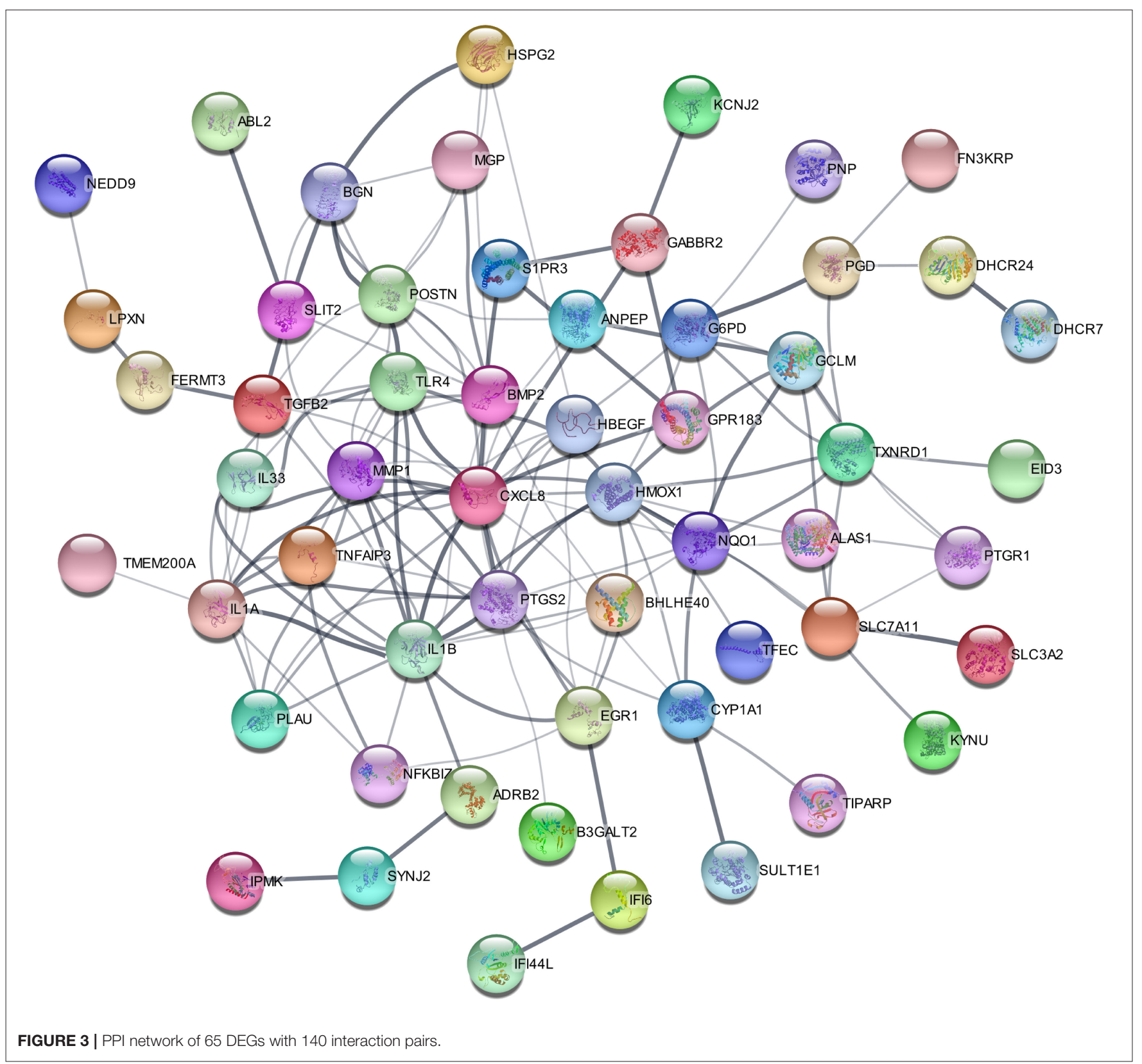

in Figure 5A. GO functional enrichment analysis suggested that 82 DEGs in the network were significantly enriched in oxidation-reduction process, angiogenesis, inflammatory response, response to hypoxia, response to oxidative stress, integral component of plasma membrane, extracellular space, cell surface, protein homodimerization activity, and transcription cofactor activity (Figures 5B-D). LncRNA n333984 and n337322 were significantly coexpressed with $M M P 1$ mRNAs in the lncRNA-mRNA coexpression network.

\section{Cis-Analyses of DELs}

To identify the cis-target genes of DELs, we searched coding genes within $10 \mathrm{~kb}$ upstream and downstream of these lncRNAs.
Cis-analyses showed that 93 DELs were adjacent to or overlapped with 60 genes in sense or antisense direction, 10 of which were differentially expressed: SESN3, SLC3A2, MGP, TLR4, TNFAIP3, MMP1, G6PD, ITGA11, CNKSR3, and TXNRD1 (Table 1).

\section{DISCUSSION}

As one of the primary supportive therapeutic methods for ARDS, mechanical ventilation could increase pulmonary microvascular permeability and inflammatory response, which further aggravates lung injury and leads to VILI (Moraes et al., 2014; Bates and Smith, 2018). The majority of "omic" studies of VILI have been carried out in lung microvascular endothelial 


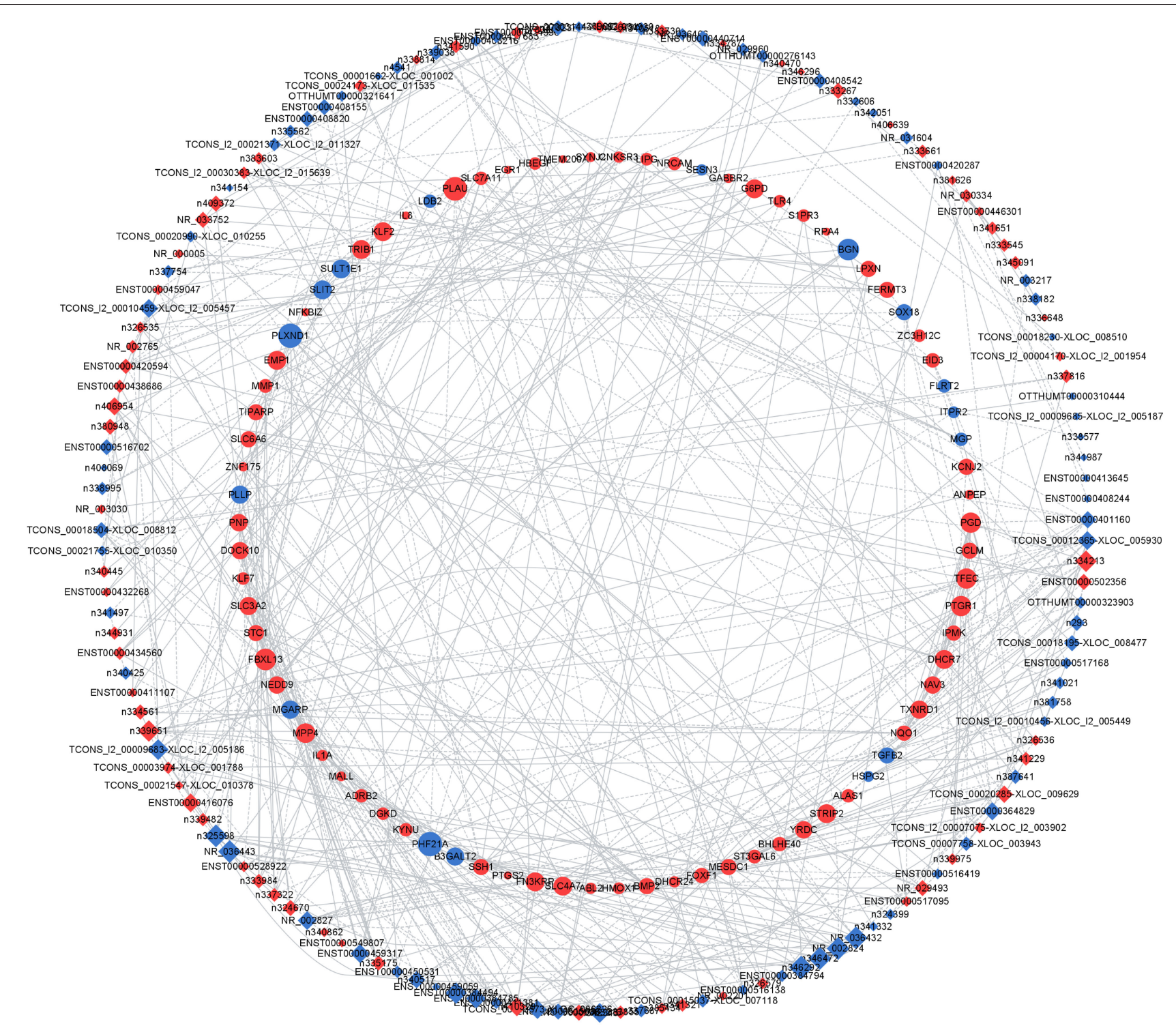

FIGURE 4 | Coexpression network of 149 DELs and 82 interacting DEGs. The diamonds represent IncRNAs; the circles represent their correlated mRNAs. Blue dots and red dots indicate downregulated and upregulated IncRNAs or mRNAs, respectively. Circle size indicates the node degree.

cells or other lung cells (Frye et al., 2005; Woods et al., 2015), or in tissues from preclinical models of VILI (Ma et al., 2005; Wang et al., 2018). To study the effects of mechanical ventilation on endothelial cell function, we stimulated HPMEC with $20 \%$ cyclic stretch for $2 \mathrm{~h}$, which was an established model of VILI to generate a mechanical-based lung injury via excessive mechanical stress (Birukov, 2009; Abiko et al., 2015; Meliton et al., 2015; Tian et al., 2016). Previously, the lncRNA expression profile was analyzed using transcriptomics of the "one-hit" mouse model of VILI (Wang et al., 2018). Different from the previous literatures, our study focused on the expression profile and transcriptional functions of endothelium-related lncRNAs associated with VILI. This is the first study to reveal the expression profile and potential roles of lncRNAs in HPMECs subjected to cyclic stretch. We have identified 199 DELs and 97 DEGs in HPMECs subjected to cyclic stretch. Integrated bioinformatics analysis demonstrated that DELs might be involved in response to hypoxia, response to oxidative stress, inflammatory response, pentose biosynthetic process, and cellular response to hypoxia. LncRNA n335470, $n 333984, n 337322$, and $n 406639$ may regulate inflammation and fibrosis induced by cyclic stretch through cis- or trans-acting mechanisms. These results provide novel perspectives of the potential molecular mechanisms underlying VILI, as well as implement fundamental evidence for future research.

Long non-coding RNA (lncRNA), whose expression is regulated by various mechanisms, such as chromatin regulation, epigenetic modifications, promoter activity modulation, and post-transcriptional mechanisms, is usually defined as transcripts 


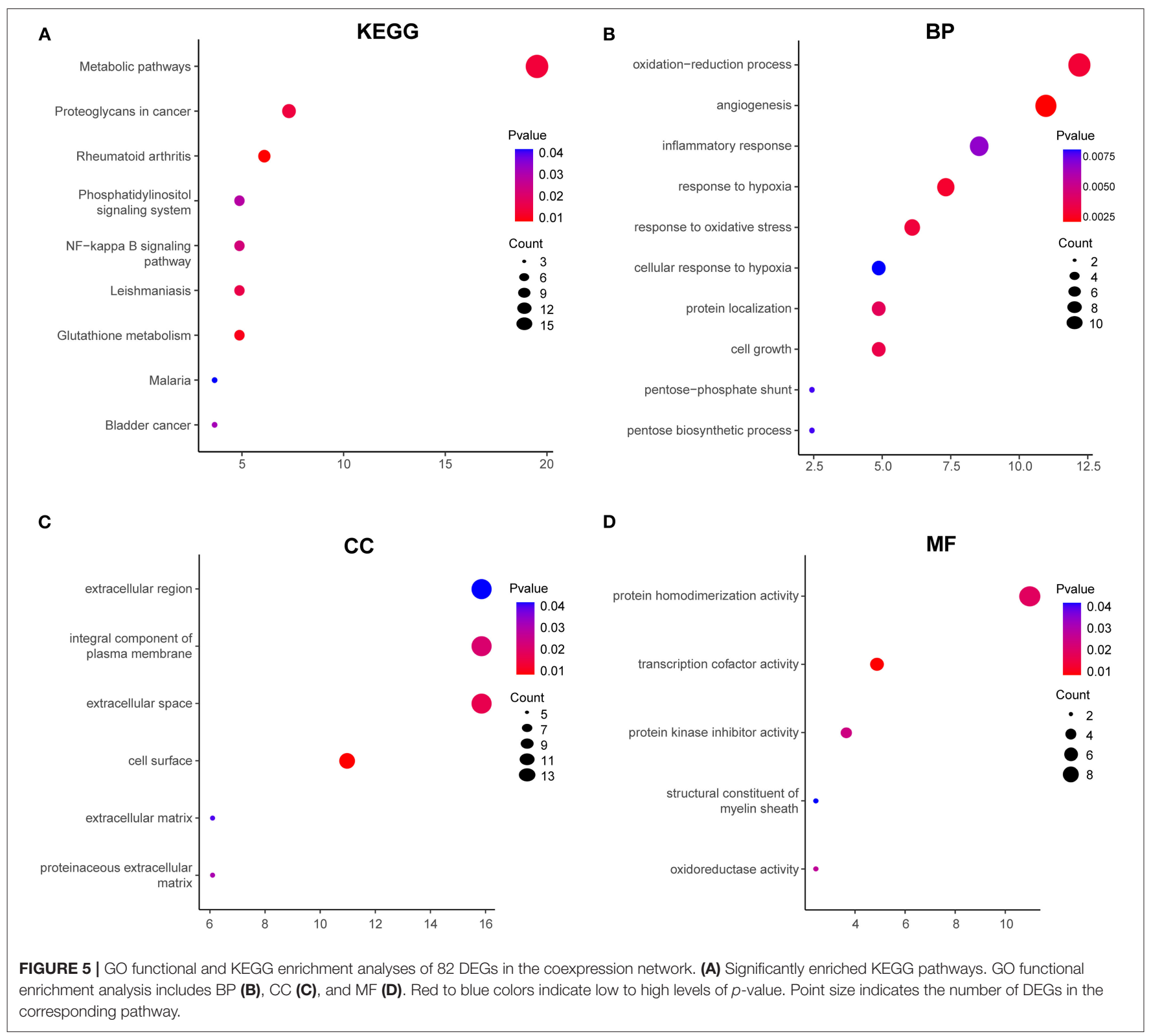

longer than 200 nucleotides with no protein-coding function (Rinn and Chang, 2012; Batista and Chang, 2013; Quinn and Chang, 2016; Tao et al., 2016). It has been reported that lncRNA could regulate gene expression in cis or trans regulatory manner (Elcheva and Spiegelman, 2020; Rinn and Chang, 2020). For cis acting manner, lncRNA loci exerts function to control the expression of neighboring or overlapping genes. This occurs through a variety of mechanisms including, cis-acting DNA-regulatory elements, the promoter region, or the act of transcription. For trans acting manner, lncRNAs regulate gene expression through binding with proteins, DNA, or other RNA, far away from the site of primary locus of transcription.

In this study, $n 335470$ and TNFAIP3 were upregulated in the HPMECs in response to mechanical ventilation, with n335470 location overlapping the TNFAIP3 gene on the same chromosome. The same pattern holds for $n 406639$ and TLR4. TNFAIP3, which is a ubiquitin-editing enzyme, could restrict inflammatory responses by negatively regulating NF$\kappa \mathrm{B}$ and MAPK signaling pathways (Yu et al., 2014). It has been reported that TNFAIP3 may stabilize the endothelial barrier by regulating the expression of VE-cadherin in acute lung injury (ALI) (Soni et al., 2018). Previous studies have also indicated that endothelial cells-expressed TLR4 increased vascular permeability and inflammatory responses in the early stages of ALI and/or ARDS (Tauseef et al., 2012; Wu et al., 2017; Peng et al., 2019). Its mechanism of action was likely to be that TLR4 induced the immune response by activating NF- $\kappa$ B signaling pathway via phosphorylating IRAK1 and IRAK2. We suggest that $n 335470$ and $n 406639$ may act as cisacting factors of TNFAIP3 and TLR4, respectively, and play 
TABLE 1 | Cis-Analysis of differentially expressed IncRNAs.

\begin{tabular}{|c|c|c|c|c|c|c|c|c|}
\hline \multicolumn{5}{|c|}{ DELs } & \multicolumn{4}{|c|}{ Cis-DEGs } \\
\hline Accession number & Database & $p$-value & Fold change & Feature & Gene symbol & $p$-value & Fold change & Feature \\
\hline n381758 & NONCODE & 0.00036 & -1.830061 & down & SESN3 & 0.002252 & -1.608661 & down \\
\hline n410329 & NONCODE & 0.00569 & 1.636504 & up & SLC3A2 & 0.013663 & 1.382277 & up \\
\hline n335562 & NONCODE & 0.006288 & -1.667897 & down & MGP & 0.016323 & -1.463823 & down \\
\hline n406639 & NONCODE & 0.014825 & 1.313714 & up & TLR4 & 0.035155 & 1.297185 & up \\
\hline n335470 & NONCODE & 0.016481 & 1.988396 & up & TNFAIP3 & 0.044094 & 1.525564 & up \\
\hline n337322 & NONCODE & 0.016943 & 1.897594 & up & MMP1 & 0.01301 & 1.87386 & up \\
\hline n334213 & NONCODE & 0.018357 & 1.521873 & up & G6PD & 0.015567 & 1.335835 & up \\
\hline n336648 & NONCODE & 0.024504 & 1.622163 & up & ITGA11 & 0.014963 & 1.496772 & up \\
\hline n344931 & NONCODE & 0.033057 & 1.348436 & up & CNKSR3 & 0.008027 & 1.550478 & up \\
\hline ENST00000549807 & ENSEMBL & 0.028716 & 1.598337 & up & TXNRD1 & 0.03917 & 1.490883 & up \\
\hline
\end{tabular}

important roles in regulation of inflammatory responses and endothelial barrier.

Our results indicated that MMP1, n333984, and $n 337322$ were significantly differentially expressed in HPMECs stimulated with mechanical ventilation, while n333984 and $n 337322$ location overlapped with MMP1 gene on the same chromosome. Moreover, lncRNA-mRNA coexpression networks analysis results showed that $n 333984$ and $n 337322$ were significantly coexpressed with MMP1 mRNA. MMP-1 played an important role in early pulmonary fibrosis through inducing the degradation of collagen type I and collagen type III (Rosas et al., 2008; Yang et al., 2020). It has been reported that MMP-1 could also decrease ROS production, and contribute to a proliferative, migratory, and anti-apoptotic phenotype, suggesting its therapeutic potential (Herrera et al., 2013). Thus, lncRNA n333984 and n337322 might be involved in fibrosis, proliferative, migratory, and anti-apoptotic by regulating the expression of MMP1 through multiple potential mechanisms.

There were several limitations of this study. First, the in vitro model had its limitations in fully reflecting biological conditions in patients, even if this VILI model was used and validated in many studies. Therefore, bias in comparison to what occurs in humans may develop. Second, our findings were mainly based on the integrated bioinformatics analysis of a relatively small sample size. Future studies containing sizable sample may be employed to validate the results both in vivo and in vitro at both protein and RNA level.

In the present study, we screened 199 DELs and explored the potential functions and underlying mechanisms of these lncRNAs through integrated bioinformatics analysis in HPMECs subjected to cyclic stretch. The DELs were mainly involved in NF-kappa B signaling pathway. LncRNA n335470, n406639, $n 333984$, and $n 337322$ might play important regulatory roles through multiple potential mechanisms. Taken together, this study provides the first transcriptomic landscape of differentially expressed lncRNAs in HPMECs subjected to cyclic stretch, which lays the groundwork for future basic and clinical research of VILI.

\section{DATA AVAILABILITY STATEMENT}

The microarray data in this study has been deposited into a publicly accessible repository (accession: GSE166772).

\section{AUTHOR CONTRIBUTIONS}

DW and CD performed bioinformatics analysis and wrote the manuscript. XZ, CG, and ML performed the experiments. HL, FY, and HW collected the data. YW reviewed and edited the manuscript. All authors contributed to the manuscript and approved the submitted version.

\section{FUNDING}

This work was supported by the National Natural Science Foundation of China (81770076, 82070078), and the Key Program of Natural Science Foundation of Shandong Province (ZR202010290035), and Academic Promotion Programme of Shandong First Medical University (2019QL015, 2019RC012), and Distinguished Taishan Scholars (ts20190981), and Young Taishan Scholars (tsqn20181244), and the Medical and Health Science and Technology Development Plan Project of Shandong Province (2019WS508).

\section{SUPPLEMENTARY MATERIAL}

The Supplementary Material for this article can be found online at: https://www.frontiersin.org/articles/10.3389/fphys. 2021.655971/full\#supplementary-material 


\section{REFERENCES}

Abiko, H., Fujiwara, S., Ohashi, K., Hiatari, R., Mashiko, T., Sakamoto, N., et al. (2015). Rho guanine nucleotide exchange factors involved in cyclic-stretchinduced reorientation of vascular endothelial cells. J. Cell Sci. 128, 1683-1695. doi: $10.1242 /$ jcs. 157503

Bader, G. D., and Hogue, C. W. (2003). An automated method for finding molecular complexes in large protein interaction networks. $B M C$ Bioinformatics 4:2. doi: 10.1186/1471-2105-4-2

Bates, J. H. T., and Smith, B. J. (2018). Ventilator-induced lung injury and lung mechanics. Ann. Transl. Med. 6:378. doi: 10.21037/atm.2018.06.29

Batista, P. J., and Chang, H. Y. (2013). Long noncoding RNAs: cellular address codes in development and disease. Cell 152, 1298-1307. doi: 10.1016/j.cell.2013.02.012

Bellani, G., Laffey, J. G., Pham, T., Fan, E., Brochard, L., Esteban, A., et al. (2016). Epidemiology, patterns of care, and mortality for patients with acute respiratory distress syndrome in intensive care units in 50 countries. JAMA 315, 788-800. doi: 10.1001/jama.2016.0291

Birukov, K. G. (2009). Cyclic stretch, reactive oxygen species, and vascular remodeling. Antioxid. Redox Signal. 11, 1651-1667. doi: 10.1089/ars.2008.2390

Chen, H., Hu, X., Li, R., Liu, B., Zheng, X., Fang, Z., et al. (2020). LncRNA THRIL aggravates sepsis-induced acute lung injury by regulating miR-424/ROCK2 axis. Mol. Immunol. 126, 111-119. doi: 10.1016/j.molimm.2020.07.021

Elcheva, I. A., and Spiegelman, V. S. (2020). The role of cis- and transacting RNA regulatory elements in leukemia. Cancers (Basel) 12:3854. doi: $10.3390 /$ cancers 12123854

Fan, E., Brodie, D., and Slutsky, A. S. (2018). Acute respiratory distress syndrome: advances in diagnosis and treatment. JAMA 319, 698-710. doi: 10.1001/jama.2017.21907

Frye, S. R., Yee, A., Eskin, S. G., Guerra, R., Cong, X., and McIntire, L. V. (2005). cDNA microarray analysis of endothelial cells subjected to cyclic mechanical strain: importance of motion control. Physiol. Genomics 21, 124-130. doi: 10.1152/physiolgenomics.00029.2003

Herrera, I., Cisneros, J., Maldonado, M., Ramirez, R., Ortiz-Quintero, B., Anso, E., et al. (2013). Matrix metalloproteinase (MMP)-1 induces lung alveolar epithelial cell migration and proliferation, protects from apoptosis, and represses mitochondrial oxygen consumption. J. Biol. Chem. 288, 25964-25975. doi: 10.1074/jbc.M113.459784

Huang da, W., Sherman, B. T., and Lempicki, R. A. (2009a). Systematic and integrative analysis of large gene lists using DAVID bioinformatics resources. Nat. Protoc. 4, 44-57. doi: 10.1038/nprot.2008.211

Huang da, W., Sherman, B. T., and Lempicki, R. A. (2009b). Bioinformatics enrichment tools: paths toward the comprehensive functional analysis of large gene lists. Nucleic Acids Res. 37, 1-13. doi: 10.1093/nar/gkn923

Huang, H., Zheng, J., Shen, N., Wang, G., Zhou, G., Fang, Y., et al. (2018). Identification of pathways and genes associated with synovitis in osteoarthritis using bioinformatics analyses. Sci. Rep. 8:10050. doi: 10.1038/s41598-018-28280-6

Jia, X., and Zhai, T. (2019). Integrated analysis of multiple microarray studies to identify novel gene signatures in non-alcoholic fatty liver disease. Front. Endocrinol. (Lausanne) 10:599. doi: 10.3389/fendo.2019.00599

Jiao, X., Sherman, B. T., Huang da, W., Stephens, R., Baseler, M. W., Lane, H. C., et al. (2012). DAVID-WS: a stateful web service to facilitate gene/protein list analysis. Bioinformatics 28, 1805-1806. doi: 10.1093/bioinformatics/bts251

Kong, F., Hu, W., Zhou, K., Wei, X., Kou, Y., You, H., et al. (2016). Hepatitis $\mathrm{B}$ virus $\mathrm{X}$ protein promotes interleukin-7 receptor expression via NFkappaB and Notchl pathway to facilitate proliferation and migration of hepatitis B virus-related hepatoma cells. J. Exp. Clin. Cancer Res. 35:172. doi: 10.1186/s13046-016-0448-2

Li, Z., Zheng, J., Xia, Q., He, X., Bao, J., Chen, Z., et al. (2020). Identification of specific long non-coding ribonucleic acid signatures and regulatory networks in prostate cancer in fine-needle aspiration biopsies. Front. Genet. 11:62. doi: $10.3389 /$ fgene.2020.00062

Liu, F., Hu, S., Zhao, N., Shao, Q., Li, Y., Jiang, R., et al. (2020). LncRNA5657 silencing alleviates sepsis-induced lung injury by suppressing the expression of spinster homology protein 2. Int. Immunopharmacol. 88:106875. doi: 10.1016/j.intimp.2020.106875
Ma, S. F., Grigoryev, D. N., Taylor, A. D., Nonas, S., Sammani, S., Ye, S. Q., et al. (2005). Bioinformatic identification of novel early stress response genes in rodent models of lung injury. Am. J. Physiol. Lung Cell. Mol. Physiol. 289, L468-L477. doi: 10.1152/ajplung.00109.2005

Matthay, M. A., Arabi, Y. M., Siegel, E. R., Ware, L. B., Bos, L. D. J., Sinha, P., et al. (2020). Phenotypes and personalized medicine in the acute respiratory distress syndrome. Intensive Care Med. 46, 2136-2152. doi: 10.1007/s00134-020-06296-9

Matthay, M. A., Zemans, R. L., Zimmerman, G. A., Arabi, Y. M., Beitler, J. R., Mercat, A., et al. (2019). Acute respiratory distress syndrome. Nat. Rev. Dis. Primers 5:18. doi: 10.1038/s41572-019-0069-0

Meliton, A., Meng, F., Tian, Y., Shah, A. A., Birukova, A. A., and Birukov, K. G. (2015). Role of krev interaction trapped-1 in prostacyclin-induced protection against lung vascular permeability induced by excessive mechanical forces and thrombin receptor activating peptide 6. Am. J. Respir. Cell Mol. Biol. 53, 834-843. doi: $10.1165 / \mathrm{rcmb} .2014-0376 \mathrm{OC}$

Moraes, L., Santos, C. L., Santos, R. S., Cruz, F. F., Saddy, F., Morales, M. M., et al. (2014). Effects of sigh during pressure control and pressure support ventilation in pulmonary and extrapulmonary mild acute lung injury. Crit. Care 18:474. doi: 10.1186/s13054-014-0474-4

Nan, C. C., Zhang, N., Cheung, K. C. P., Zhang, H. D., Li, W., Hong, C. Y., et al. (2020). Knockdown of IncRNA MALAT1 alleviates LPS-induced acute lung injury via inhibiting apoptosis through the miR-194-5p/FOXP2 axis. Front. Cell Dev. Biol. 8:586869. doi: 10.3389/fcell.2020.586869

Otasek, D., Morris, J. H., Boucas, J., Pico, A. R., and Demchak, B. (2019). Cytoscape automation: empowering workflow-based network analysis. Genome Biol. 20:185. doi: 10.1186/s13059-019-1758-4

Peng, L. Y., Yuan, M., Shi, H. T., Li, J. H., Song, K., Huang, J. N., et al. (2019). Protective effect of piceatannol against acute lung injury through protecting the integrity of air-blood barrier and modulating the TLR4/NF-kappaB signaling pathway activation. Front. Pharmacol. 10:1613. doi: 10.3389/fphar.2019.01613

Pham, T., and Rubenfeld, G. D. (2017). Fifty years of research in ARDS. the epidemiology of acute respiratory distress syndrome. a 50th birthday review. Am. J. Respir. Crit. Care Med. 195, 860-870. doi: 10.1164/rccm.201609-1773CP

Quinn, J. J., and Chang, H. Y. (2016). Unique features of long non-coding RNA biogenesis and function. Nat. Rev. Genet. 17, 47-62. doi: 10.1038/nrg.2015.10

Rinn, J. L., and Chang, H. Y. (2012). Genome regulation by long noncoding RNAs. Annu. Rev. Biochem. 81, 145-66. doi: 10.1146/annurev-biochem-051410-092902

Rinn, J. L., and Chang, H. Y. (2020). Long noncoding RNAs: molecular modalities to organismal functions. Annu. Rev. Biochem. 89, 283-308. doi: 10.1146/annurev-biochem-062917-012708

Rosas, I. O., Richards, T. J., Konishi, K., Zhang, Y., Gibson, K., Lokshin, A. E., et al. (2008). MMP1 and MMP7 as potential peripheral blood biomarkers in idiopathic pulmonary fibrosis. PLoS Med. 5:e93. doi: 10.1371/journal.pmed.0050093

Shannon, P., Markiel, A., Ozier, O., Baliga, N. S., Wang, J. T., Ramage, D., et al. (2003). Cytoscape: a software environment for integrated models of biomolecular interaction networks. Genome Res. 13, 2498-2504. doi: $10.1101 /$ gr. 1239303

Soni, D., Wang, D. M., Regmi, S. C., Mittal, M., Vogel, S. M., Schluter, D., et al. (2018). Deubiquitinase function of A20 maintains and repairs endothelial barrier after lung vascular injury. Cell Death Discov. 4:60. doi: 10.1038/s41420-018-0056-3

Szklarczyk, D., Gable, A. L., Lyon, D., Junge, A., Wyder, S., Huerta-Cepas, J., et al. (2019). STRING v11: protein-protein association networks with increased coverage, supporting functional discovery in genome-wide experimental datasets. Nucleic Acids Res. 47, D607-D613. doi: 10.1093/nar/gky1131

Tao, Z., Yuan, Y., and Liao, Q. (2016). Alleviation of lipopolysaccharidesinduced acute lung injury by MiR-454. Cell. Physiol. Biochem. 38, 65-74. doi: $10.1159 / 000438609$

Tauseef, M., Knezevic, N., Chava, K. R., Smith, M., Sukriti, S., Gianaris, N., et al. (2012). TLR4 activation of TRPC6-dependent calcium signaling mediates endotoxin-induced lung vascular permeability and inflammation. J. Exp. Med. 209, 1953-1968. doi: 10.1084/jem.20111355

Tian, Y., Gawlak, G., O’Donnell, J. J. III, Birukova, A. A., and Birukov, K. G. (2016). Activation of Vascular Endothelial Growth Factor (VEGF) receptor 2 
mediates endothelial permeability caused by cyclic stretch. J. Biol. Chem. 291, 10032-10045. doi: 10.1074/jbc.M115.690487

Wang, H. R., Guo, X. Y., Liu, X. Y., and Song, X. (2020). Downregulation of lncRNA CASC9 aggravates sepsis-induced acute lung injury by regulating miR-195-5p/PDK4 axis. Inflamm. Res. 69, 559-568. doi: 10.1007/s00011-020-01316-2

Wang, L., Zhang, N., Zhang, Y., Xia, J., Zhan, Q., and Wang, C. (2018). Landscape of transcription and long non-coding RNAs reveals new insights into the inflammatory and fibrotic response following ventilator-induced lung injury. Respir. Res. 19:122. doi: 10.1186/s12931-018-0822-z

Woods, S. J., Waite, A. A., O’Dea, K. P., Halford, P., Takata, M., and Wilson, M. R. (2015). Kinetic profiling of in vivo lung cellular inflammatory responses to mechanical ventilation. Am. J. Physiol. Lung Cell. Mol. Physiol. 308, L912-L921. doi: 10.1152/ajplung.00048.2015

Wu, H., Yang, Y., Guo, S., Yang, J., Jiang, K., Zhao, G., et al. (2017). Nuciferine ameliorates inflammatory responses by inhibiting the TLR4-mediated pathway in lipopolysaccharide-induced acute lung injury. Front. Pharmacol. 8:939. doi: 10.3389/fphar.2017.00939

Yang, H., Hua, C., Yang, X., Fan, X., Song, H., Peng, L., et al. (2020). Pterostilbene prevents LPS-induced early pulmonary fibrosis by suppressing oxidative stress, inflammation and apoptosis in vivo. Food Funct. 11, 4471-4484. doi: 10.1039/C9FO02521A

Yu, W. W., Lu, Z., Zhang, H., Kang, Y. H., Mao, Y., Wang, H. H., et al. (2014). Antiinflammatory and protective properties of daphnetin in endotoxin-induced lung injury. J. Agric. Food Chem. 62, 12315-12325. doi: 10.1021/jf503667v
Yu, Y., Sun, H., Zhu, L., Ji, L., and Liu, H. (2020). Downregulating lncRNA PRNCR1 ameliorates LPS-induced pulmonary vascular endothelial cell injury by modulating miR-330-5p/TLR4 axis. J. Biochem. Mol. Toxicol. 35:e22644. doi: $10.1002 /$ jbt. 22644

Zhang, X., Chen, K., Chen, X., Kourkoumelis, N., Li, G., Wang, B., et al. (2020). Integrative analysis of genomics and transcriptome data to identify regulation networks in female osteoporosis. Front. Genet. 11:600097. doi: 10.3389/fgene.2020.600097

Zhou, H., Wang, X., and Zhang, B. (2020). Depression of lncRNA NEAT1 antagonizes LPS-evoked acute injury and inflammatory response in alveolar epithelial cells via HMGB1-RAGE signaling. Mediators Inflamm. 2020:8019467. doi: 10.1155/2020/ 8019467

Conflict of Interest: The authors declare that the research was conducted in the absence of any commercial or financial relationships that could be construed as a potential conflict of interest.

Copyright (C) 2021 Wang, Dai, Zhang, Gu, Liu, Liu, Yang, Wu and Wang. This is an open-access article distributed under the terms of the Creative Commons Attribution License (CC BY). The use, distribution or reproduction in other forums is permitted, provided the original author(s) and the copyright owner(s) are credited and that the original publication in this journal is cited, in accordance with accepted academic practice. No use, distribution or reproduction is permitted which does not comply with these terms. 